\title{
Endoscopy in Pregnancy
}

\author{
Paul Mitrut, Anca Oana Docea, \\ Cornelia - Daniela Calina and Liliana Streba \\ Additional information is available at the end of the chapter \\ http://dx.doi.org/10.5772/52550
}

\section{Introduction}

In most of gastrointestinal disorders, endoscopy has a major diagnostic and therapeutic role, but though its clinical efficacy and safety have been established, it is not that well known when performing it in pregnant patients, due to the potential harm of the foetus like hypoxia, teratogenesis, trauma, placental abruption or induction of premature labor.

Endoscopy is generally considered to be a low risk procedure, the most frequently being performed at the patient's request in ambulatory but also in hospitals [1]. Nevertheless, the safety and effectiveness of gastrointestinal endoscopy in particular circumstances at the pregnant woman has not been yet well studied. During the pregnancy the risks for the fetus and mother are various and the magnitude of this risk is different, according to the trimester. Taking into consideration the difficulties in the performance of those studies to pregnant women these risks have not been enough evaluated.

The main concern is the fetal safety and endoscopic medication and use of sedative and analgesics represent a significant risk if they are not chosen properly. It is strongly indicated the presence of an anesthesiologist and an obstetrician, in selected cases when a pregnancyrelated complication is most probable. Before the procedure, the pregnant patient should be well evaluated by the gastroenterologist, anesthesiologist and obstetrician and should be informed about the potential risks of sedation and analgesia.

Because of the fact that there are potential risks for the fetus and for the pregnant woman, the indications of endoscopy to pregnant women limit to superior gastrointestinal hemorrhages, dysphagia, uncontrolled nausea/vomits, rectal bleeding, diarrhea, biliary lithiasis or biliary pancreatitis [2]. For that matter, endoscopy for pregnant women is considered to be a very rare procedure; in USA only 19000 pregnant women do this type of annual investigation [3]. 
It is being performed during the pregnancy only when there are no other ways of diagnosis or therapy less invasive, the indications for the procedure are clear and as much as possible the gastroscopy should be postponed until third trimester.

The safety of gastrointestinal endoscopy during the pregnancy was evaluated by Cappell et al [4] who performed esophago-gastro-duodenoscopies on a lot of 83 pregnant women and concluded on the fact that the procedure is safe and does not induce birth or congenital malformations. The same authors investigated the safety of sigmoidscopy to 46 patients and established the safety of the procedure to the pregnant women who had small gastrointestinal bleeding and avoided to induce early labor and the appearance of congenital malformations. Nevertheless, as a result of data which are rather limited and incomplete in what concerns the safety of the procedure, fetal risk to endoscopy in the pregnancy has not been completely excluded. In that effect, in Qureshi 's work et al. which has been approved by the American Society of Endoscopic Gastrointestinal Endoscopy (ASGE, 2005) there had been distinguished the principles that the doctor must comply with when he decides to make endoscopies to a pregnant woman [5].

\section{Physiological modifications in the pregnancy and the endoscopy}

Pregnant women are susceptible to gastrointestinal reflux disease, biliary tract complications and gastrointestinal bleeding, thus needing to be performed upper endoscopy, ERCP or colonoscopy. For example, only in the United States, upper endoscopy was necessary in over 12,000 pregnant patients per year and colonoscopy or sigmoidoscopy in more than 6000 pregnant patients per year. ERCP is also needed, as cholelithiasis has a $12 \%$ incidence in pregnant patients. Latest studies suggest that, if having good indication (upper gastrointestinal bleeding, refractory nausea or vomiting), upper endoscopy is relatively safe in pregnant patients.

Increase in size of pregnant uterus determines the lift of stomach along with the modification of intra abdominal segment of esophagus that gets into the thorax. As a result it is being reduced the tonus of the inferior esophagian sphincter and increases intragastric pressure which predisposes to gastroesophageal reflux. Even if gastric volume and acidity of gastric juice does not modify during the pregnancy, the pregnant women show a regurgitating risk through the decrease of pressure barrier of inferior eshophageal sphincter. This risk justifies the pharmacological methods of reduction of gastric secretion. $50-80 \%$ from the pregnant women show "retrosternal burning-pyrosis" which is the clinical correspondent of gastroesophageal reflux. On those grounds, the pregnant women are considered as having a full stomach [6].

During the last months of pregnancy, the pregnant uterus modifies anatomic relations between abdominal and pelvic organs. These modifications can extend the time of performance of the procedure, they can increase the quantity of anaesthesic medicines that are being administered and the air quantity inoculated in order to facilitate the intraluminal visualization. In order to diminish the compression of vena cava by the pregnant uterus there should 
be adopted the lying down position of the pregnant woman, position that was used in a normal way for colonoscopy. For those grounds, the endoscopist must be accustomed with endoscopic procedures to pregnant women and fully evaluate anatomic and pathological modifications of superior and interior gastrointestinal tract.

Biliary oversaturation and biliary hypomotility are as well frequently seen as physiological modifications that appear during the pregnancy. Unfortunately, this can lead to the formation of biliary sludge and biliary calculi, which at their turn can widen biliary colics, cholecystitis and even pancreatitis.

Airway mucosa is edematiated during the pregnancy, showing a reduction of airways. Moreover, there is a compensatory increase of ventilation on each minute in order to answer to demands of maternal and fetal oxygen. Nevertheless, pulmonary capacity is reduced as a result of ascension of the diaphragm.

As well, during the pregnancy there appear hemodynamic modifications. Cardiac frequency increases from 90 to 100 beats a minute. Systolic arterial pressure can increase. Pregnant women cannot often tolerate the supine position, especially after 30 pregnancy weeks, given reduced arterial pressure because of big weight of the uterus.

\section{Endoscopy indications during pregnancy}

Endoscopy should be performed during pregnancy only when the indication for the procedure is clear and there are no less invasive or therapeutical diagnosis ways. In order to perform this operation it is necessary the patient's informed consent. It should be taken into account that the procedure should be postponed until second trimester. The specific indications for endoscopy during pregnancy, as ASGE recommends are the following:

- Significant or continued GI bleeding

- Severe or refractory nausea and vomiting or abdominal pain

- Dysphagia or odynophagia

- Strong suspicion of colon mass

- Severe diarrhea with negative evaluation

- Biliary pancreatitis, choledocholithiasis, or cholangitis

- Biliary or pancreatic ductal injury

Also, there are a few principles (ASGE) to respect:

- have a strong indication

- preferably perform endoscopy during the second trimester of pregnancy

- use lowest effective dose of sedative drugs and of category A or B, if available 
- minimize procedure time

- position pregnant patients in left pelvic tilt or left lateral position to avoid vena cava or aortic compression

- presence of fetal heart sounds should be confirmed before sedation is begun and after the endoscopic procedure

- obstetric support should be available in the event of a pregnancy-related complication

- endoscopy is contraindicated in obstetric complications such as placental abruption, imminent delivery, ruptured membranes, or eclampsia.

The decision of performing an endoscopy to a pregnant patient must be taken by a team: obstetrician - endoscopist - anaesthesist, because there must be analyzed: the implications for the fetus, for the mother, the emergency of the situation and the possibility of therapeutical alternatives in order to solve the issue in safety conditions or postponement after labor [7]. An obstetrician must be available during the procedure if there are complications related to the pregnancy.

\section{Fetal and maternal risks in endoscopic explorations}

\subsection{Endoscopic risks for the fetus}

They can be: hypoxy given to oversedation, fetal hyperperfusion given to maternal position, teratogenity given to the medicines administration, uterine trauma along with the impact on the fetus through endoscopic trauma, preterm labor through uterine compression. That is why it is advisable to avoid the performance of endoscopies in first trimester, excepting clinical emergency cases.

Among these risks, potential risk to dermine a malformation in fetal development through pills administration, early labor or giving mechanical uterine trauma seriously need evaluation when it is being analyzed an endoscopic procedure to pregnant people. Moreover, medical and ethical problems require a reticence for doctors and pregnant people in what concerns endoscopic studies and require the patient's informed consent. Fetal normal status must be confirmed before starting the endoscopy and it must be revaluated as soon as possible after its completion.

There is no evidence that certifies increased fetal morbidity for pregnant women that are subject to endoscopic procedures in comparison with pregnant women that had not been subject at any investigations. On the contrary, a case control study that enclosed a number of 83 superior digestive endoscopies performed to pregnant women showed that there had not been any preterm labors and any new born children of these women had not different Apgar scores to birth in comparison with those women that had not performed these kind of investigations [4].

A survey performed by 300 specialist gastroenterologists doctors, which included information regarding 73 digestive superior endoscopies and 13 colonoscopies performed during 
the pregnancy, did not succeed to report any significant complication for those pregnancies [8]. Just as well, a series of groups from 10 medical centers reported an experience of 48 flexible sigmoidoscopies and 8 colonoscopies performed during the pregnancy [9] and dit not report any negative result that could affect the fetus and that could be assigned to those endoscopic procedures.

From the point of view of gestation development, fetal complications are: fetal abnormalities, labor induction, preterm labor that could be determined by endoscopic procedures, having the greatest risk to appear in the first trimester of pregnancy [10]. In general, the second trimester is considered to have the lowest risk for endoscopic interventions.

Even if the studies that had been performed did not seem to indicate a negative result for the fetus and which could have been assigned directly to endoscopy, the procedure itself requires a great caution from the doctors. Endoscopy, which has a diagnostic or therapeutical role must be taken into consideration only when, without its execution, the risks are higher. General rules are to postpone the procedure until labor or at least after first trimester of pregnancy. Pre-procedure training of the pregnant woman must include a special examination from the obstetrician and his availability in care of eventual complications. There will be monitored the fetal heart beats before starting the procedure that will be revaluated as soon as possible after the completion.

The risk of fetal hypoxy can be reduced through minimum sedation of the pregnant woman. The procedure is not performed with the mother in position of lying down, because the pregnant uterus can compress the aorta and/inferior vena cava that causes maternal hypotension and therefore it could interfere with the placental perfusion. Therefore it is advisable left lateral position. American Society for Gastrointestinal Endoscopy has recently published the guidelines that must be fulfilled for the endoscopy to pregnant women, based on available data and the consent of specialist doctors [11].

Fetal monitoring is made through the hearts beats with the help of a monitor and it allows to the doctor to detect any fatal suffering. This difficulty can be improved through the correction of maternal hypoxy or hypotension.

In 2009 ASA and American College of obstetrics and gynecology (ACOG) issued a common statement regarding the endoscopic interventions performed during pregnancy [12]. The document contains the following mentions:

- endoscopist must obtain a pre-operative examination together with the obstetrician before performing each endoscopic procedure. This must be achieved no matter the gestation age of the fetus.

- when the fetus is pre-viable, Doppler detection of fetal heart beats immediately before and after the procedure is enough.

- when the fetus is viable, continuous intra-operative monitoring of fetal heart beats and the presence of a gynecology surgeon is ideal, if it appears an emergency labor.

The decision to use a monitor for fetal heart beats should be individualized, according to the availability of resources. 


\subsection{Endoscopic risks for the pregnant woman}

Endoscopic risks for the pregnant woman can be:

- precipitated aspiration through abdominal dystensia

- hypotension through the compression of inferior vena cava

- uterine trauma through the anatomic modification

Maternal hypotension appears through the compression of inferior vena cava and the reduction of venous return. That is why there must be avoided the supine position during the procedures.

Compression of abdominal organs by the pregnant uterus can modify the digestive lumen and therefore the duration of endoscopy can be extended, and for the vizualization it is necessary a higher amount of air breathed, the endoscope can jump-up (especially to colonoscopies) and it can appear an abdominal dystensia accompanied by a discomfort of the pregnant woman.

Endoscopy for pregnant women is made only in carefully selected cases. It can't be made an endoscopy in case of an imminent abortion or if there are some obstetrical problems. The procedure is better performed during day in a specialized endoscopy office. It can be administered oxygen, but it is not compulsory.

\section{Sedative medication used for endoscopy in pregnancy}

As well the mother and the fetus are subject to some potential risks in case of endoscopic sedation. These risks vary according to pregnancy trimester.

Sedative and analgesic agents should always be used in that smaller dose in order to minimize the potential risk of teratogenic effects. The highest risk for the fetus takes place during the first trimester when it is the most vulnerable to possible teratogenic effects.

As there are no well controlled studies regarding the safety of the fetus towards the pharmacological agents that are used for sedation in the pregnant women endoscopy, no medicine has been framed by FDA in class A, without teratogenity [13].

Data for safe medicines, not teratogenic, are reduced, because the clinical studies to pregnant women are very rarely performed, they are expensive and require long term monitoring [14]. This point of view led to the recommendation of sedative medicines for endoscopy during the pregnancy in very small quantities and only if it is strictly necessary.

A good cooperation between the pregnant woman and gastroenterologist should bring to the performance of a fast endoscopic procedure, simple and without sedation. But most of them are made in emergency situations and with therapeutical intentions. In these situations it is necessary the sedation, and the endoscopist should consult with obstetrician doctor and with anasthesist about the anesthesic medicines, taking into account the FDA classification 
of teratogenity of medicines. It is very important that the doctor be familiar with the stages of fetal development and teratogenesis. At two weeks from the conception, embrionary cells are subject to the law "all or nothing". In this period the exposure to toxic drugs will lead either to a normal and healthy fetus or the embryo will not be viable. In the next eight weeks and until the second trimester it appears the difference of cells and organogenesis. At the moment, the exposure to teratogenic medicines will have as a result severe congenital malformations. In the second and third trimester the medicines can still generate fetal toxicity, especially from the neurological point of view.

In order to classify their safety when using in pregnancy, drugs were divided by FDA into 5 categories:

1. category A - defined as adequate and well controlled studied in pregnant women;

2. category B - animal studies have revealed no evidence of harm to the fetus, but there are no adequate studies in pregnant women or animal studies have shown an adverse effect, but adequate studies in humans have failed to demonstrate a risk to the fetus;

3. category $\mathrm{C}$ - animal studies have shown an adverse effect, and there are no adequate studies in pregnant women or no animal studies have been conducted and there are no adequate studies in pregnant women;

4. category D - studies in pregnant women have demonstrated a risk to the fetus (however, the benefits of therapy may outweigh the risk);

5. category $X$ - studies in both animals and humans have demonstrated evidence of fetal abnormalities; use is contraindicated in women who are or may be pregnant.

Because there are no well controlled studies regarding fetus safety when using pregnant patients sedation in endoscopy, no drug was classified as category A by the FDA.

Most of the drugs used in pregnant patients sedation are category B or C. Category D drugs should be avoided and used only when the benefit outweighs the risks, while category $\mathrm{X}$ it is not used at all. Sedation should be moderate or anxiolisis; in case of deep sedation is needed, it should be only under the surveillance of a specialised anesthesiologist. It should be used the lowest efficacious dose of sedation or, if possible, the endoscopy should be performed without any sedation: most upper endoscopies and sigmoidoscopies can be accomplished without sedation.

The most common medicines that are used for endoscopic sedation and analgesia are Meperidine, Fentanyl, benzodiazepines and Propofol. Meperidine (FDA category B) does not seem to be teratogenic. It is better than choosing Fentanyl during pregnancy. Fentanyl (FDA category C) as well, can be relatively safe in small doses, but there are few available data referring to its safety. The use of some benzodiazepines (FDA category D) is not yet controversial.

The use of Diazepam is not recommended during pregnancy. The use of Diazepam during pregnancy had been associated with split of the hard palate to new born infants, according to some old studies [15] but other two meta-analyses did not succeed to confirm 
these results [16]. Midazolam has not been associated with fetal malformations, but its use must be avoided in the first trimester. The safety of Propofol (FDA category B) has not yet been established, but taking into account the short duration of action, with minimum secondary effects, it could be a drug of choice when it is necessary a more profound sedation.

In the first trimester endoscopic procedures must rather be achieved without sedation or by using Meperidine all alone. In the second and third trimester Meperidine remains the first choice drug, but little doses of Midazolam can be added according to needs.

If it is necessary a more profound sedation, it is recommended the examination with the anaesthesist. The pregnancy in trimesters two and three represents a great challenge for endoscopies, taking into account the management of airways, sedation and monitoring cardiac and respiratory functions and the fluids balance. Approaching must be made by a team that includes the obstetrician, anaesthesist and the endoscopist working together in a hospital.

There are certain drugs commonly used in gastrointestinal endoscopy: meperidine, fentanyl, naloxone, benzodiazepines, pethidine, flumazenil, propofol, simethicone, glucagon, topical anesthetics, colon-cleansing agents that need to be discussed extensively, according to their category.

\subsection{Meperidine (category B)}

Of all opioidagonists that have teratogenic effects on animals, meperidine appears to be safe when being used for endoscopy in pregnant women[17; 18]. This is one of the standard drugs used for analgesia and sedation and is the preferred opiate during pregnancy. It does not cross the blood brain barriers or a pidasmorphineandis often used by obstetricians for analgesia during labor. However, using high doses close to birth time, may cause neonatal respiratory depression. Meperidine may also be responsible for transient fetal heart rate abnormalities, but in the absence of other fetal changes it can not be considered an indicator of poor prognosis in this situation[19].

To obtain the minimum sedation effect during endoscopy in pregnant women it should be used the lowest dose, limited to a maximum of $75 \mathrm{mg}$ meperidine during routine exploration. In case of occasional occurrence of respiratory depression or hypotension secondary to opiate use, there can be used rapid acting opiate antagonists such as naloxone (B category) [20]. It crosses the placenta shortly after administered to the mother and was not shown to be associated with teratogenicity. It is preferred compared to fentanyl and morphine.

\subsection{Fentanyl (category C)}

It is considered that fentanyl is a safe opiate when used in low doses if administered during pregnancy. It has a faster onset of action than meperidine and is generally indicated in pregnant patients with a previous history of seizures. Although it was found as being embryocidal to rats, clinical experience in pregnant women was very similar to meperidine [21]. 


\subsection{Naloxone (category B)}

It doesn't seem to be teratogenic. It is contraindicatedin mothers who are dependent on opiates because it may precipitate opiate-withdrawal symptoms. It is being used only in cases of respiratory depression, hypotension, or lack of response and under strict monitoring. It should be noted that there is a risk of resedation due to metabolising the drug.

\subsection{Flumazenil (category C)}

It is a benzodiazepine antagonist, not very well studied, but it seems it might determine neurobehavioral changes in rats if exposed to it in utero.

\subsection{Benzodiazepines (category D)}

Diazepam accumulates rapidly into fetal circulation after maternal administration and was associated with congenital defects in mice. As concerning the occurrence of similar anomalies in humans, opinions are divided. The data suggest that administration of diazepam in early pregnancy appears to be a risk factor, although using it during the second and third trimester could theoretically determine neurobehavioral disorders in neonates [25]. Therefore, the conclusion is that diazepam should not be used during pregnancy.

Midazolam is the most commonly used sedative for endoscopic procedures. Although it crosses the placentaandcanbedetected to the fetus, unlike diazepam, it is not concentrated in the fetal circulation and was not associated with congenital defects. However, belonging to benzodiazepines, it has the neonatal respiratory depressant potential. Therefore it should be used in small doses, with great care under the supervision of ananesthesiologist, when sedation with meperidineisin sufficient [26].A meta-analysis of studies on the risks of anesthesia for gastroscopies during pregnancy concluded that the only potential problem is a slight increased incidence of abortion in the first or second trimester of pregnancy[27]. It is not recommended during the first trimester of pregnancy [28].

\subsection{Propofol (category B)}

Propofolis an increasingly used for endoscopy sedative drug, especially in the United States. It is short acting, with a much shorter recovery period than other sedative pharmacological agents[22]. However, following an arrow therapeutic index and potential respiratory depression, itis generally administered only by anesthesiologists. Gastroenterologists' medical societies have recommended that this agent should be reserved for deeper sedation and other complicated clinical situations in a highly monitored for vital functions environment [23]. It is considered relatively safe for use during pregnancy, although few data are available regarding its use in the first trimester of pregnancy[24].

\subsection{Simethicone (category C)}

It is frequently used in pregnant patients, with no reported addverse effects, but it is not extensively studied yet so it belongs to category $\mathrm{C}$ drugs. 


\subsection{Glucagon (category B)}

It is a safe to use antispasmodic, especially in ERCP.

\subsection{Topical anesthetics (category B)}

Lidocaine is used to provide pharingeal anesthesia and it was reported as being safe to use even in the first trimester of pregnancy.

\subsection{Colon cleansing-agents}

Most of them are category $\mathrm{C}$, due to lack of studies during pregnancy, and this is the case for PEG solutions (polyethylene glycol) and sodium phosphate solutions which can cause fluid disturbance. It is considered that for flexible sigmoidoscopy tap water enemas are sufficient.

\section{Endoscopy and variceal bleeding in pregnancy}

Variceal gastrointestinal bleedings during pregnancy are true emergencies. A delay in their treatment could lead to serious complications and even death of the mother and fetus. Therapeutic endoscopic procedures (sclerotherapy) are recommended as first line treatment. Effectiveness and relative safety of these approaches during pregnancy is documented in the literature [29]. Octreotide is used to treat variceal bleeding but its safety in pregnancy has not been established.Non-variceal gastrointestinal bleeding were successfully treated with endoscopic therapy during pregnancy using an epinephrine injection plus either placement of hemostatic clips or thermo-coagulation.

\section{Endoscopic Retrograde Cholangiopancreatography (ERCP)}

It is strongly recommended to be performed by very well trained endoscopists and is indicated in pregnant patients with pancreatitis, cholangitis, choledocholithiasis (complicated with jaundice, impacted or not). If possible, ERCP should be postponed from the first trimester to the second in order to minimize irradiation's potential teratogenic effect. There should be used a lead shielding for the patient's abdomen, a guidewire as opposed to injection of contrast and minimal use of fluoroscopy and spot radiographs.

\section{Lower endoscopy}

It is indicated in important lower intestinal bleeding, severe unexplained diarrhea and colon neoplasm suspicion. Colorectal cancer screening or change in bowel habits are indications that may be postponed for the postpartum period.

Most of the studies specify sigmoidoscopy as a safely procedure during pregnancy (in stable pregnant patients, having strong indication: sigmoid or rectal mass, severe diarrhea prolonged and not responding to specific treatment, lower intestinal hemorrhage). It should be postponed until after birth for patients complaining abdominal pain or change in bowel 
habits. Sigmoidoscopy is not causing congenital abnormalities and isn't inducing labor, all studies performed so far reporting delivery of healthy new borns at term.

Colonoscopy safety during pregnancy is not yet properly studied, thus guidelines are not available. It is recommended only for life-threatening situations. Maneuvers like placing the patient in the prone position or exerting external abdominal compression should be avoided, especially in the third trimester of pregnancy.

\section{Nonendoscopic imaging modalities of the digestive tract during pregnancy}

There can be used endoscopic videocapsules which offer theoretical advantages of gastrointestinal mucosa inspection. They do not need sedation of the patient or mechanical pressure on the abdomen, but safety of their use during pregnancy is unknown. Originally designed to view the small intestine, a change in the original design is now available to inspect the esophagus. It remains to be seen if technical adjustments of this technology will allow future use for the colonas well.

Virtual colonoscopy by MRI is safer during pregnancy than using classic CT. Although still classified as "experimental", virtual technologies are increasingly used as a screening tool for colorectal neoplasia. However, its safety has not been studied in pregnancy although there are some reports showing the usefulness and lack of teratogenicity from abdominal MRI with gadolinium as contrast agent in different clinical situations during pregnancy $[30 ; 31 ; 32]$.

\section{Conclusions}

Performing endoscopies in pregnant patients has not only to be done by a physician experienced in general gastroenterology and endoscopic procedures but also highly experienced in performing such procedures in pregnant women. It is not recommended for these endoscopies to be performed by a beginner, as they carry the unique issue of fetal safety. Endoscopic interventions in pregnancy must be performed quickly and with caution. The endoscopist should always be prepared to discontinue the action at any time for safety reasons. An anesthesiologist and an obstetrician must be part of the working team, especially ready for emergencies.

A number of studies concluded that esophagogastroduodenoscopy and sigmoidoscopy are not contraindicated during pregnancy. If for instance significant upper gastrointestinal bleeding is suspected, emergency esophagogastroduodenoscopy should be performed. Sigmoidoscopy is to be considered when the pregnant patient is stabilized, and only if strong indications for this procedure exist. 
Even if no definitive data exist at the present time, ERCP should be performed when the possibility for a sphincterotomy exists. It should only be attempted by highly trained personnel, in a center with extensive experience and the resources to resolve all possible issues that may incur.

\section{Author details}

Paul Mitrut ${ }^{1}$, Anca Oana Docea ${ }^{2}$, Cornelia - Daniela Calina ${ }^{2}$ and Liliana Streba ${ }^{*}$

${ }^{*}$ Address all correspondence to: lilianastreba@gmail.com

1Department of Internal Medicine, University of Medicine and Pharmacy of Craiova, Romania

2 Department of Pharmacology, University of Medicine and Pharmacy of Craiova, Romania

\section{References}

[1] Mitruț P., Mitrut Anca Oana, Streba Liliana, Calina Daniela, Salplahta D. Sedation related to gastrointestinal endoscopy. In: Pascu O. (ed.) Gastrointestinal Endoscopy. InTech, 2011; 3: 23-44.

[2] O'MahonyS. Endoscopy in pregnancy. Best Pract Res ClinGastroenterol. 2007; 21: 893-899.

[3] Cappell MS. The fetal safety and clinical efficacy of gastrointestinal endoscopy during pregnancy. GastroenterolClin North A m 2003; 32: 123-179.

[4] Cappell MS, Colon VJ, SidhomOA . A study of eight medical centers of the safety and clinical efficacy of esophagogastroduodenoscopy in 83 pregnant females with follow up of fetal outcome with comparison control groups. A m J Gastroenterol 1996; 9: 348-354.

[5] Qureshi WA, Rajan E, Adler DG, Davila RE, Hirota WK, Jacobson BC, Leighton JA, Zuckerman MJ, Hambrick RD, Fanelli RD, Baron T, Faigel DO. American Society for Gastrointestinal Endoscopy. ASGE Guideline: Guidelines for endoscopy in pregnant and lactating women. Gastrointest Endosc 2005; 6: 357-362.

[6] Acalovschi I, Anestezie clinică, Ed. Clusium Cluj Napoca, 2001.

[7] Gilinsky NH, Muthunayagam N. Gastrointestinal endoscopy in pregnant and lactating women: emerging standard of care to guide decision-making. Obstetrical \& Gynecological Survey 2006; 61:791-799. 
[8] Frank B. Endoscopy in pregnancy. In: Karlstadt RG, Surawicz CM, Croitoru R, eds. Gastrointestinal Disorders During Pregnancy. American College of Gastroenterology, 1994: 24-29

[9] Cappell MS, Colon VJ, SidhomOA . A study at 10 medical centers of the safety and efficacy of 48 flexible sigmoidoscopies and 8 colonoscopies during pregnancy with follow-up of fetal outcome and with comparison to control groups. Dig Dis Sci 1996; 4: 2353-2361.

[10] Gupta R, Tandan M, Lakhtakia S, Santosh D, Rao GV, Reddy DN. Safety of therapeutic ERCP in pregnancy-an Indian experience. Indian J Gastroenterol 2005; 24: 161-163.

[11] American Society for Gastrointestinal Endoscopy. Guidelines for Endoscopy in Pregnant and Lactating Women. Gastrointestinal Endoscopy. Vol. 61, No. 3: 2005.

[12] American Society of Anesthesiologists (ASA) and the American College of Obstetricians and Gynecologists (ACOG) Statement on non obstetric surgery during pregnancy $10 / 2009$.

[13] Meadows M. Pregnancy and the drug dilemma. FDA Consumer Magazine, MayJune 2001. Available at: www.fda.gov/fdac/features/2001/301_preg.html.

[14] Powrie RO. Principles for drug prescribing in pregnancy. Editors: Rosene-Montella K., Keely E., Barbour LA., Lee RV. Medical care of the pregnant patient. 2nd ed. Philadelphia: ACP press. 2008.

[15] Safra MJ., Okley GP., Association between cleft lip with or without cleft palate and prenatal exposure to Diazepam. Lancet 1975; 2: 478-80.

[16] Dolovich LR., Addis A., Vaillancourt JM., et al. Benzodiazepine use in pregnancy and major malformations or oral cleft: metaanalysis of cohort and case-control studies. BMJ 1998; 317: 839-43.

[17] Briggs GG, Freeman RK, Yaffe SJ. Drugs in Pregnancy and Lactation: A Reference Guide to Fetal and Neonatal Risk, 7th ed. Philadelphia: Lippincott Williams \& Wilkins, 2005.

[18] Briggs GG, Wan SR. Drug therapy during labor and delivery, part 1. Am J Health Syst Pharm 2006; 63: 1038-1047.

[19] Cunningham FG, Grant NF, Leveno KJ, et al. Analgesia and sedation. In: Cunningham FG, Grant NF, Leveno KJ, et al, eds. William's Obstetrics, 21st Ed. New York: McGraw-Hill, 2001: 537-563.

[20] Cappell MS. The fetal safety and clinical efficacy of gastrointestinal endoscopy during pregnancy. GastroenterolClin N Am 2003. 32: 123-179.

[21] Martin LV, Jurand A. The absence of teratogenic effects of some analgesics used in anesthesia: additional evidence from the mouse model. Anesthesia 1992. 47: 473-6.

[22] Lazear SE, Course 1055: Moderate sedation/analgesia. CME resources. P. 44. 
[23] Augustyn D, Brill JV, Faigel D, et al. National Affairs: Three Gastroenterology Specialty Groups Issue Joint Statement on Sedation in Endoscopy. March 8, 2004. Available at: www.acg.gi.org/members/nataffairs/trisociety.asp.

[24] Gin T. Propofol during pregnancy. ActaAnaesthesiol Sin 1994;32:127-132.

[25] Ornoy A, Arnon J, Shechtman S, et al. Is benzodiazepine use during pregnancy really teratogenic? ReprodToxicol 1998; 12: 511-515.

[26] Hawkins JL. Anesthesia for the pregnant patient undergoing nonobstetric surgery. ASA 2005; 33: 137-144.

[27] Boiven JF. Risk of spontaneous abortion in women occupationally exposed to anesthetic gases: A meta analysis. OccupEnviron Med 1997; 54: 541-548.

[28] Kost M. Moderate Sedation/Analgesia: Core Competencies for Practice, 2nd Ed. St. Louis, MO: Saunders, St. Louis; 2004; 59.

[29] Starkel P., Horsmans Y., Geubel A. Endoscopic band ligation: a safe technique to control bleeding esophageal varices in pregnancy. GastrointestEndosc1998; 48: 212-214.

[30] Brown MA, Birchard KR, Seminelka RC. Magnetic resonance evaluation of pregnant patients with acute abdominal pain. Semin Ultrasound CT MR 2005; 26: 206-211.

[31] Pedrosa I, Levine D, Eyvazzadeh AD. MR imaging evaluation of acute appendicitis in pregnancy. Radiology 2006; 238: 891-899.

[32] Garcia-Bournissen F, Shrim A, Koren G. Safety of gadolinium during pregnancy. Can Fam Physician 2006; 52: 309-310. 